\title{
High-Order Random Walks and Generalized Laplacians on Hypergraphs
}

\author{
Linyuan Lu and Xing Peng
}

Abstract. Despite the extreme success of spectral graph theory, there are relatively few papers applying spectral analysis to hypergraphs. Chung first introduced Laplacians for regular hypergraphs and showed some useful applications. Other researchers have treated hypergraphs as weighted graphs and then studied the Laplacians of the corresponding weighted graphs. In this paper, we aim to unify these very different versions of Laplacians for hypergraphs. We introduce a set of Laplacians for hypergraphs through studying high-order random walks on hypergraphs. We prove that the eigenvalues of these Laplacians can effectively control the mixing rate of high-order random walks, the generalized distances/diameters, and the edge expansions.

\section{Introduction}

Many complex networks (such as spatial networks [Demir et al. 08], cellular networks [Klamt et al. 09], and biomolecular networks [Zhang 07]) have richer structures than graphs can have. Inherently, they have hypergraph structures: interconnections often cross multiple nodes. Treating these networks as graphs causes a loss of structure. Nonetheless, it is still popular to use graph tools to

(C) Taylor \& Francis Group, LLC

ISSN: I542-795I print 
study these networks; one of them is the Laplacian spectrum. Let $G$ be a graph on $n$ vertices. The Laplacian $\mathcal{L}$ of $G$ is the $n \times n$ matrix $I-T^{-1 / 2} A T^{-1 / 2}$, where $A$ is the adjacency matrix and $T$ is the diagonal matrix of degrees. Let $\lambda_{0}, \lambda_{1}, \ldots, \lambda_{n-1}$ be the eigenvalues of $\mathcal{L}$, indexed in nondecreasing order. It is known that $0 \leq \lambda_{i} \leq 2$ for $0 \leq i \leq n-1$. If $G$ is connected, then $\lambda_{1}>0$. The first nonzero Laplacian eigenvalue $\lambda_{1}$ is related to many graph parameters, such as the mixing rate of random walks, the graph diameter, the neighborhood expansion, the Cheeger constant, the isoperimetric inequalities, expander graphs, and quasirandom graphs [Aldous and Fill 12, Alon 86, Chung 89, Chung et al. 94, Chung 97, Lawler and Sokal 88, Mihail 89].

In this paper, we define a set of Laplacians for hypergraphs. Laplacians for regular hypergraphs were first introduced in [Chung 93], which used the homology approach. The first nonzero Laplacian eigenvalue can be used to derive several useful isoperimetric inequalities. It seems hard to extend Chung's definition to general hypergraphs. There are some researchers who have treated a hypergraph as a multiedge graph and then defined its Laplacian to be the Laplacian of a corresponding multiedge graph. For example, it was shown in [Rodríguez 09] that the approach above has some applications to bisections, the average minimal cut, the isoperimetric number, the max-cut, the independence number, the diameter, etc. Rodríguez's approach was introduced in [Li 04]; Ramanujan hypergraphs were defined in [Li and Solé 96]. Another direction for studying the spectra of adjacency matrices of hypergraphs is to use the notion of tensor product. This direction was taken in [Friedman and Wigderson 95] to investigate spectra of adjacency matrices of 3-uniform hypergraphs. Recently, spectra of adjacency hypermatrices of $r$-uniform hypergraphs were considered in [Cooper and Dutle 12].

What are the "right" Laplacians for hypergraphs? To answer this question, let us recall how the Laplacian was introduced in graph theory. One approach uses a geometric/homological analogue, where the Laplacian is defined as a selfadjoint operator on the functions over vertices. Another approach uses random walks, where the Laplacian is the symmetrization of the transition matrix of the random walk on a graph. In [Chung 93], the first approach was taken, and Chung defined her Laplacians for regular hypergraphs. In this paper, we take the second approach and define the Laplacians through high-order random walks on hypergraphs.

A high-order walk on a hypergraph $H$ can be roughly viewed as a sequence of overlapped oriented edges $F_{1}, F_{2}, \ldots, F_{k}$. For $1 \leq s \leq r-1$, we say that $F_{1}, F_{2}, \ldots, F_{k}$ is an $s$-walk if $\left|F_{i} \cap F_{i+1}\right|=s$ for each $i$ in $\{1,2,3, \ldots, k-1\}$. For example, a Hamiltonian $s$-cycle is a special $s$-walk that covers each vertex exactly once. There are many papers [Dudek and Frieze 11, Dudek and Frieze 12, Frieze 10, Hàn and Schacht 99, Katona and Kierstead 99, Keevash 
et al. 12, Kühn et al. 12, Kühn and Osthus 06, Rödl et al. 06, Rödl et al. 08] studying Hamiltonian s-cycles in hypergraphs. A detailed definition of high-order random walks will be given later.

From this rough view of high-order random walks, if we refer to the intersection of two consecutive edges as a stop, then it is possible that there is some vertex from the stop contained in more than two consecutive edges when $s>r / 2$, so we need to specify the edge to which the vertex from the stop belongs. Hence we need to assume a stop to be an ordered set of $s$ vertices, which causes an $s$-walk to be reversible when $1 \leq s \leq r / 2$ and irreversible when $s>r / 2$. Therefore, in the definition of the $s$ th Laplacian for $r$-uniform hypergraphs, we will split the definition into two cases. The loose case is that in which $1 \leq s \leq r / 2$; in this case we will reduce the $s$ th Laplacian of a hypergraph to the Laplacian of an associated weighted undirected graph. The tight case is that in which $r / 2<s \leq r-1$; in this case we will reduce the sth Laplacian of a hypergraph to the Laplacian of an associated Eulerian directed graph.

Before we give the definition of the sth Laplacian of a hypergraph, we need to review necessary results on the Laplacian of a weighted undirected graph and the Laplacian of an Eulerian directed graph. The choice of $s$ enables us to define a set of Laplacian matrices $\mathcal{L}^{(s)}$ for $H$. For $s=1$, our definition of Laplacian $\mathcal{L}^{(1)}$ is the same as the definition in [Rodríguez 09]. For $s=r-1$, while we restrict our attention to regular hypergraphs, our definition of the Laplacian $\mathcal{L}^{(r-1)}$ is similar to the definition in [Chung 93]. We will discuss the relationship between these notions in the final section of this paper. Our definition of Laplacians is also closely related to the singular values used in the unpublished work [Butler unpubl.].

In this paper, we show several applications of the Laplacians of hypergraphs, such as the mixing rate of high-order random walks, generalized diameters, and edge expansions. Our approach allows users to select a "right" Laplacian to fit their special requirements.

The rest of the paper is organized as follows. In Section 2, we review and prove some useful results on the Laplacians of weighted graphs and Eulerian directed graphs. The definition of Laplacians for hypergraphs will be given in Section 3 . We will prove some properties of the Laplacians of hypergraphs in Section 4, and consider several applications in Section 5. In the final section, we will comment on future directions.

\section{Preliminary Results}

In this section, we review some results on Laplacians of weighted graphs and Eulerian directed graphs. The results on Laplacians of weighted graphs will be applied 
to the $s$ th Laplacians of hypergraphs for $1 \leq s \leq r / 2$, and those on Laplacians of Eulerian directed graphs will be applied to the $s$ th Laplacians of hypergraphs for $r / 2<s \leq r-1$.

In this paper, we frequently switch domains from hypergraphs to weighted (undirected) graphs and/or to directed graphs. To reduce confusion, we use the following conventions throughout this paper. We denote a weighted graph by $G$, a directed graph by $D$, and a hypergraph by $H$. The sets of vertices are denoted by $V(G), V(D)$, and $V(H)$, respectively. (Whenever it is clear from the context, we will write such a set as $V$ for short.) The edge sets are denoted by $E(G), E(D)$, and $E(H)$, respectively. The degrees $d_{*}$ and volumes vol $(*)$ are defined separately for the weighted graph $G$, for the directed graph $D$, and for the hypergraph $H$. Readers are warned to interpret them carefully depending on the context.

For a positive integer $s$ and a vertex set $V$, let $\mathrm{V}^{\underline{s}}$ be the set of all (ordered) $s$-tuples consisting of $s$ distinct elements in $V$. Let $\left(\begin{array}{c}V \\ s\end{array}\right)$ be the set of all unordered (distinct) $s$-subsets of $V$.

Let 1 be the row (or the column) vector with all entries of value 1, and let $I$ be the identity matrix. For a row (or column) vector $f$, the norm $\|f\|$ is always the $L_{2}$-norm of $f$.

\section{I. Laplacians of Weighted Graphs}

A weighted graph $G$ on the vertex set $V$ is an undirected graph associated with a weight function $w: V \times V \rightarrow \mathbb{R} \geq 0$ satisfying $w(u, v)=w(v, u)$ for all $u$ and $v$ in $V(G)$. Here we always assume $w(v, v)=0$ for every $v \in V$.

A simple graph can be viewed as a special weighted graph such that each edge has weight 1 and each non-edge has weight 0 . Many concepts of simple graphs are naturally generalized to weighted graphs. If $w(u, v)>0$, then $u$ and $v$ are adjacent, written as $x \sim y$. The graph distance $d(u, v)$ between two vertices $u$ and $v$ in $G$ is the minimum integer $k$ such that there is a path $u=v_{0}, v_{1}, \ldots, v_{k}=v$ in which $w\left(v_{i-1}, v_{i}\right)>0$ for $1 \leq i \leq k$. If no such $k$ exists, then we let $d(u, v)=\infty$. If the distance $d(u, v)$ is finite for every pair $(u, v)$, then $G$ is connected. For a connected weighted graph $G$, the diameter (denoted by $\operatorname{diam}(G)$ ) is the largest value of $d(u, v)$ among all pairs of vertices $(u, v)$.

The adjacency matrix $A$ of $G$ is defined as the matrix of weights, i.e., $A(x, y)=$ $w(x, y)$ for all $x$ and $y$ in $V$. The degree $d_{x}$ of a vertex $x$ is $\sum_{y} w(x, y)$. Let $T$ be the diagonal matrix of degrees in $G$. The Laplacian $\mathcal{L}$ is the matrix $I-T^{-1 / 2} A T^{-1 / 2}$. Let $\lambda_{0}, \lambda_{1}, \ldots, \lambda_{n-1}$ be the eigenvalues of $\mathcal{L}$, indexed in nondecreasing order. It is known [Chung 97] that $0 \leq \lambda_{i} \leq 2$ for $0 \leq i \leq n-1$. If $G$ is connected, then $\lambda_{1}>0$. 
From now on, we assume that $G$ is connected. The first nontrivial Laplacian eigenvalue $\lambda_{1}$ is the most useful one. It can be written in terms of the Rayleigh quotient as follows (see [Chung 97]):

$$
\lambda_{1}=\inf _{f \perp T} \frac{\sum_{x \sim y}(f(x)-f(y))^{2} w(x, y)}{\sum_{x} f(x)^{2} d_{x}} .
$$

Here the infimum is taken over all functions $f: V \rightarrow \mathbb{R}$, which is orthogonal to the degree vector $\mathbf{1} T=\left(d_{1}, d_{2}, \ldots, d_{n}\right)$. Similarly, the largest Laplacian eigenvalue $\lambda_{n-1}$ can be defined in terms of the Rayleigh quotient as follows:

$$
\lambda_{n-1}=\sup _{f \perp T \mathbf{1}} \frac{\sum_{x \sim y}(f(x)-f(y))^{2} w(x, y)}{\sum_{x} f(x)^{2} d_{x}} .
$$

Note that scaling the weights by a constant factor will not affect the Laplacian. A weighted graph $G$ is complete if $w(u, v)=c$ for some constant $c$ such that $c>0$, independent of the choice of $(u, v)$ with $u \neq v$. We say that $G$ is bipartite if there is a partition $V=L \cup R$ such that $w(x, y)=0$ for all $x, y \in L$ and all $x, y \in R$.

We have the following facts (see [Chung 97]):

1. $0 \leq \lambda_{i} \leq 2$ for each $0 \leq i \leq n-1$.

2. The number of 0 eigenvalues equals the number of connected components in $G$. If $G$ is connected, then $\lambda_{1}>0$.

3. $\lambda_{n-1}=2$ if and only if $G$ has a connected component that is a bipartite weighted subgraph.

4. $\lambda_{n-1}=\lambda_{1}$ if and only if $G$ is a complete weighted graph.

It turns out that $\lambda_{1}$ and $\lambda_{n-1}$ are related to many graph parameters, such as the mixing rate of random walks, the diameter, the edge expansions, and the isoperimetric inequalities.

A random walk on a weighted graph $G$ is a sequence of vertices $v_{0}, v_{1}, \ldots, v_{k}$ such that the conditional probability $\operatorname{Pr}\left(v_{i+1}=v \mid v_{i}=u\right)$ is equal to $w(u, v) / d_{u}$ for $0 \leq i \leq k-1$. A vertex probability distribution is a map $f: V \rightarrow \mathbb{R}$ such that $f(v) \geq 0$ for each $v$ in $G$ and $\sum_{v \in V} f(v)=1$. It is convenient to write a vertex probability distribution as a row vector. A random walk maps a vertex probability distribution to a vertex probability distribution through multiplying by a transition matrix $P$ from the right, where $P(u, v)=w(u, v) / d_{u}$ for each pair of vertices $u$ and $v$. We can write $P=T^{-1} A=T^{-1 / 2}(I-\mathcal{L}) T^{1 / 2}$. The spectral gap $\bar{\lambda}(P)$, denoted by $\bar{\lambda}$ for short, is $\max \left\{\left|1-\lambda_{1}\right|,\left|1-\lambda_{n-1}\right|\right\}$. Let $\pi(u)=d_{u} / \operatorname{vol}(G)$ for each vertex $u$ in $G$. Observe that $\pi$ is the stationary distribution of the random walk, i.e., $\pi P=\pi$. A random walk is mixing if $\lim _{i \rightarrow \infty} f_{0} P^{i}=\pi$ for every initial vertex probability distribution $f_{0}$. It is known that a random walk is always 
mixing if $G$ is connected and not a bipartite graph. To overcome the difficulty resulting from being a bipartite graph (where $\lambda_{n-1}=2$ ) for $0 \leq \alpha \leq 1$, we consider an $\alpha$-lazy random walk, whose transition matrix $P_{\alpha}$ is given by $P_{\alpha}(u, u)=\alpha$ for each $u$ and $P_{\alpha}(u, v)=(1-\alpha) w(u, v) / d_{u}$ for each pair of vertices $u$ and $v$ with $u \neq v$. Note that the transition matrix is

$$
P_{\alpha}=\alpha I+(1-\alpha) T^{-1} A=T^{-1 / 2}(I-(1-\alpha) \mathcal{L}) T^{1 / 2} .
$$

Let

$$
L_{\alpha}=T^{1 / 2} P_{\alpha} T^{-1 / 2}=I-(1-\alpha) \mathcal{L}
$$

and

$$
\bar{\lambda}_{\alpha}=\max \left\{\left|1-(1-\alpha) \lambda_{1}\right|,\left|1-(1-\alpha) \lambda_{n-1}\right|\right\}
$$

Since $L_{\alpha}$ is a symmetric matrix, we have

$$
\bar{\lambda}_{\alpha}=\max _{u \perp T^{1 / 2} 1} \frac{\left\|L_{\alpha} u\right\|}{\|u\|} .
$$

It turns out that the mixing rate of an $\alpha$-lazy random walk is determined by $\bar{\lambda}_{\alpha}$.

Theorem 2.I. For $0 \leq \alpha \leq 1$, the vertex probability distribution $f_{k}$ of the $\alpha$-lazy random walk at time $k$ converges to the stationary distribution $\pi$ in probability. In particular, we have

$$
\left\|\left(f_{k}-\pi\right) T^{-1 / 2}\right\| \leq \bar{\lambda}^{k}\left\|\left(f_{0}-\pi\right) T^{-1 / 2}\right\| .
$$

Here $f_{0}$ is the initial vertex probability distribution.

Proof. Notice that $f_{k}=f_{0} P_{\alpha}^{k}$ and $\left(f_{0}-\pi\right) T^{-1 / 2} \perp 1 T^{1 / 2}$. We have

$$
\begin{aligned}
\left\|\left(f_{k}-\pi\right) T^{-1 / 2}\right\| & =\left\|\left(f_{0} P_{\alpha}^{k}-\pi P_{\alpha}^{k}\right) T^{-1 / 2}\right\|=\left\|\left(f_{0}-\pi\right) P_{\alpha}^{k} T^{-1 / 2}\right\| \\
& =\left\|\left(f_{0}-\pi\right) T^{-1 / 2} L_{\alpha}^{k}\right\| \leq \bar{\lambda}_{\alpha}^{k}\left\|\left(f_{0}-\pi\right) T^{-1 / 2}\right\| .
\end{aligned}
$$

This completes the proof.

For each subset $X$ of $V(G)$, the volume $\operatorname{vol}(X)$ is $\sum_{x \in X} d_{x}$. If $X=V(G)$, then we write $\operatorname{vol}(G)$ instead of $\operatorname{vol}(V(G))$. We have

$$
\operatorname{vol}(G)=\sum_{i=1}^{n} d_{i}=2 \sum_{u \sim v} w(u, v) .
$$


If $\bar{X}$ is the complement of $X$, then we have $\operatorname{vol}(\bar{X})=\operatorname{vol}(G)-\operatorname{vol}(X)$. For every pair of subsets $X$ and $Y$ of $V(G)$, the distance $d(X, Y)$ between $X$ and $Y$ is $\min \{d(x, y): x \in X, y \in Y\}$.

Theorem 2.2. [Chung 89, Chung 97] In a weighted graph $G$, for $X, Y \subseteq V(G)$ with distance at least 2 , we have

$$
d(X, Y) \leq\left\lceil\log \sqrt{\frac{\operatorname{vol}(\bar{X}) \operatorname{vol}(\bar{Y})}{\operatorname{vol}(X) \operatorname{vol}(Y)}} / \log \frac{\lambda_{n-1}+\lambda_{1}}{\lambda_{n-1}-\lambda_{1}}\right\rceil .
$$

A special case of Theorem 2.2 is that both $X$ and $Y$ are single vertices, which gives an upper bound on the diameter of $G$.

Corollary 2.3. [Chung 97] If $G$ is not a complete weighted graph, then we have

$$
\operatorname{diam}(G) \leq\left\lceil\log \frac{\operatorname{vol}(G)}{\delta} / \log \frac{\lambda_{n-1}+\lambda_{1}}{\lambda_{n-1}-\lambda_{1}}\right\rceil
$$

where $\delta$ is the minimum degree of $G$.

For $X, Y \subseteq V(G)$, let $E(X, Y)$ be the set of edges between $X$ and $Y$. Namely, we have

$$
E(X, Y)=\{(u, v): u \in X, v \in Y \text { and } u v \in E(G)\} .
$$

We have the following theorem.

Theorem 2.4. [Chung 89, Chung 97] If $X$ and $Y$ are two subsets of $V(G)$, then we have

$$
|| E(X, Y)\left|-\frac{\operatorname{vol}(X) \operatorname{vol}(Y)}{\operatorname{vol}(G)}\right| \leq \bar{\lambda} \cdot \frac{\sqrt{\operatorname{vol}(X) \operatorname{vol}(Y) \operatorname{vol}(\bar{X}) \operatorname{vol}(\bar{Y})}}{\operatorname{vol}(G)}
$$

\subsection{Laplacians of Eulerian Directed Graphs}

The Laplacian of a general directed graph was introduced in [Chung 05, Chung 06]. The theory is considerably more complicated than the one for undirected graphs, but when we consider a special class of directed graphs, namely Eulerian directed graphs, it turns out to be quite neat.

Let $D$ be a directed graph with vertex set $V(D)$ and edge set $E(D)$. A directed edge from $x$ to $y$ is denoted by an ordered pair $(x, y)$ or by $x \rightarrow y$. The out-neighborhood $\Gamma^{+}(x)$ of a vertex $x$ in $D$ is the set $\{y:(x, y) \in E(D)\}$. The 
out-degree $d_{x}^{+}$is $\left|\Gamma^{+}(x)\right|$. Similarly, the in-neighborhood $\Gamma^{-}(x)$ is $\{y:(y, x) \in$ $E(D)\}$, and the in-degree $d_{x}^{-}$is $\left|\Gamma^{-}(x)\right|$. A directed graph $D$ is Eulerian if $d_{x}^{+}=d_{x}^{-}$ for every vertex $x$. In this case, we simply write $d_{x}=d_{x}^{+}=d_{x}^{-}$for each $x$. For a vertex subset $S$, the volume of $S$, denoted by $\operatorname{vol}(S)$, is $\sum_{x \in S} d_{x}$. In particular, we write $\operatorname{vol}(D)=\sum_{x \in V} d_{x}$.

Eulerian directed graphs have many nice properties. For example, an Eulerian directed graph is strongly connected if and only if it is weakly connected.

The adjacency matrix of $D$ is a square matrix $A$ satisfying $A(x, y)=1$ if $(x, y) \in E(D)$ and $A(x, y)=0$ otherwise. Let $T$ be the diagonal matrix with $T(x, x)=d_{x}$ for each $x \in V(D)$. Let $\overrightarrow{\mathcal{L}}=I-T^{-1 / 2} A T^{-1 / 2}$, i.e.,

$$
\overrightarrow{\mathcal{L}}(x, y)= \begin{cases}1 & \text { if } x=y \\ -1 / \sqrt{d_{x} d_{y}} & \text { if } x \rightarrow y \\ 0 & \text { otherwise }\end{cases}
$$

Note that $\overrightarrow{\mathcal{L}}$ is not symmetric. We define the Laplacian $\mathcal{L}$ of $D$ to be the symmetrization of $\overrightarrow{\mathcal{L}}$, that is,

$$
\mathcal{L}=\frac{\overrightarrow{\mathcal{L}}+\overrightarrow{\mathcal{L}^{\prime}}}{2}
$$

Since $\mathcal{L}$ is symmetric, its eigenvalues are real and can be listed as $\lambda_{0}, \lambda_{1}, \ldots, \lambda_{n-1}$ in nondecreasing order. Note that $\lambda_{1}$ can also be written in terms of the Rayleigh quotient (see [Chung 05]) as follows:

$$
\lambda_{1}=\inf _{f \perp T \mathbf{1}} \frac{\sum_{x \rightarrow y}(f(x)-f(y))^{2}}{2 \sum_{x} f(x)^{2} d_{x}} .
$$

In [Chung 06], there is a general theorem on the relationship between $\lambda_{1}$ and the diameter. After restricting to Eulerian directed graphs, it can be stated as follows.

Theorem 2.5. [Chung 06] Suppose D is a connected Eulerian directed graph. Then the diameter of $D$ satisfies

$$
\operatorname{diam}(D) \leq\left\lfloor\frac{2 \log (\operatorname{vol}(G) / \delta)}{\log \frac{2}{2-\lambda_{1}}}\right\rfloor+1,
$$

where $\lambda_{1}$ is the first nontrivial eigenvalue of the Laplacian matrix, and $\delta$ is the minimum degree $\min \left\{d_{x} \mid x \in V(D)\right\}$.

The main idea in the proof of this theorem is to use $\alpha$-lazy random walks on $D$. A random walk on an Eulerian directed graph $D$ is a sequence of vertices 
$v_{0}, v_{1}, \ldots, v_{k}$ such that for $0 \leq i \leq k-1$, the conditional probability $\operatorname{Pr}\left(v_{i+1}=\right.$ $v \mid v_{i}=u$ ) equals $1 / d_{u}$ for each $v \in \Gamma^{+}(u)$ and is 0 otherwise. For $0 \leq \alpha \leq 1, \alpha-$ lazy random walks are defined similarly. The transition matrix $P_{\alpha}$ of an $\alpha$-lazy random walk satisfies

$$
P_{\alpha}=\alpha I+(1-\alpha) T^{-1} A=T^{-1 / 2}(I-(1-\alpha) \overrightarrow{\mathcal{L}}) T^{1 / 2} .
$$

In [Chung 05], only 1/2-lazy random walks are considered. Here we prove some results on $\alpha$-lazy random walks for $\alpha \in[0,1)$.

Let $\pi(u)=d_{u} / \operatorname{vol}(D)$ for each $u \in V(D)$. Note that $\pi$ is the stationary distribution, i.e., $\pi P_{\alpha}=\pi$. Let

$$
L_{\alpha}=\alpha I+(1-\alpha) T^{-1 / 2} A T^{-1 / 2}=I-(1-\alpha) \overrightarrow{\mathcal{L}}=T^{1 / 2} P_{\alpha} T^{-1 / 2} .
$$

The key observation is that there is a unit vector $\phi_{0}$ such that $\phi_{0}$ is a row eigenvector $L_{\alpha}$ and $\phi_{0}^{T}$ is a column eigenvector of $L_{\alpha}$ for the largest eigenvalue 1. Here let

$$
\phi_{0}=\mathbf{1} \cdot \frac{T^{1 / 2}}{\operatorname{vol}(D)}=\frac{1}{\operatorname{vol}(G)}\left(\sqrt{d_{1}}, \ldots, \sqrt{d_{n}}\right) .
$$

We have

$$
\phi_{0} L_{\alpha}=\phi_{0} \quad \text { and } \quad L_{\alpha} \phi_{0}^{\prime}=\phi_{0}^{\prime} .
$$

Let $\phi_{0}^{\perp}$ be the orthogonal complement of $\phi_{0}$ in $R^{n}$. It is easy to check that $L_{\alpha}$ maps $\phi_{0}^{\perp}$ to $\phi_{0}^{\perp}$. Let $\sigma_{\alpha}$ be the spectral norm of $L_{\alpha}$ when restricted to $\phi_{0}^{\perp}$. An equivalent definition of $\sigma_{\alpha}$ is the second-largest singular value of $L_{\alpha}$, i.e.,

$$
\sigma_{\alpha}=\max _{f \perp \phi_{0}^{\prime}} \frac{\left\|L_{\alpha} f\right\|}{\|f\|} .
$$

Lemma 2.6. We have the following properties for $\sigma_{\alpha}$ :

1. For every $\beta \in \phi_{0}^{\perp}$, we have $\left\|L_{\alpha} \beta\right\| \leq \sigma_{\alpha}\|\beta\|$.

2. $\left(1-\lambda_{1}\right)^{2} \leq \sigma_{0}^{2} \leq 1$.

3. $\sigma_{\alpha}^{2} \leq \alpha^{2}+2 \alpha(1-\alpha) \lambda_{1}+(1-\alpha)^{2} \sigma_{0}^{2}$.

Proof. Item 1 is from the definition of $\sigma_{\alpha}$. Since the largest eigenvalue of $L_{\alpha}$ is 1 , we have $\sigma_{\alpha} \leq 1$. In particular, $\sigma_{0}^{2} \leq 1$. Note that $L_{0}=T^{-1 / 2} A T^{-1 / 2}$. Let $f=g T^{1 / 2}$. It follows that

$$
\sigma_{0}^{2}=\sup _{f \perp \phi_{0}^{\prime}} \frac{\left\|L_{0} f\right\|^{2}}{\|f\|^{2}}=\sup _{g \perp T 1} \frac{g^{\prime} A^{\prime} T^{-1} A g}{g^{\prime} T g} .
$$


Choose $g \in(T \mathbf{1})^{\perp}$ such that the Rayleigh quotient (2.4) reaches its minimum at $g$, i.e.,

$$
\lambda_{1}=\frac{\sum_{x \rightarrow y}(g(x)-g(y))^{2}}{2 \sum_{x} g(x)^{2} d_{x}} .
$$

We have

$$
\begin{aligned}
\frac{g^{\prime} A^{\prime} T^{-1} A g}{g^{\prime} T g} & =\frac{\sum_{x} \frac{1}{d_{x}}\left(\sum_{y \in \Gamma^{+}(x)} g(y)\right)^{2}}{\sum_{x} d_{x} g(x)^{2}} \\
& =\frac{\sum_{x} d_{x} g(x)^{2} \sum_{x} \frac{1}{d_{x}}\left(\sum_{y \in \Gamma^{+}(x)} g(y)\right)^{2}}{\left(\sum_{x} d_{x} g(x)^{2}\right)^{2}} \\
& \geq \frac{\left(\sum_{x} g(x) \sum_{y \in \Gamma^{+}(x)} g(y)\right)^{2}}{\left(\sum_{x} d_{x} g(x)^{2}\right)^{2}} \\
& =\left(\frac{\sum_{x} g(x) \sum_{y \in \Gamma^{+}(x)} g(y)}{\sum_{x} d_{x} g(x)^{2}}\right)^{2}=\left(1-\lambda_{1}\right)^{2}
\end{aligned}
$$

In the last step, we have used the following argument:

$$
\begin{aligned}
\frac{\sum_{x} g(x) \sum_{y \in \Gamma^{+}(x)} g(y)}{\sum_{x} d_{x} g(x)^{2}} & =\frac{\frac{1}{2} \sum_{x \rightarrow y}\left(g(x)^{2}+g(y)^{2}-(g(x)-g(y))^{2}\right)}{\sum_{x} d_{x} g(x)^{2}} \\
& =1-\frac{\sum_{x \rightarrow y}(g(x)-g(y))^{2}}{2 \sum_{x} d_{x} g(x)^{2}}=1-\lambda_{1} .
\end{aligned}
$$

Since $\sigma_{0}$ is the maximum over all $g \perp T \mathbf{1}$, we get $\left(1-\lambda_{1}\right)^{2} \leq \sigma_{0}^{2}$.

For item 3, we have

$$
\begin{aligned}
\sigma_{\alpha}^{2} & =\sup _{f \perp \phi_{0}^{\prime}} \frac{\left\|L_{\alpha} f\right\|^{2}}{\|f\|^{2}}=\sup _{g \perp T \mathbf{1}} \frac{g^{\prime} P_{\alpha}^{\prime} T P_{\alpha} g}{g^{\prime} T g} \\
& \leq \alpha^{2}+\alpha(1-\alpha) \sup _{g \perp T \mathbf{1}} \frac{g^{\prime}\left(A+A^{\prime}\right) g}{g^{\prime} T g}+(1-\alpha)^{2} \sup _{g \perp T \mathbf{1}} \frac{g^{\prime} A^{\prime} T^{-1} A g}{g^{\prime} T g} \\
& =\alpha^{2}+2 \alpha(1-\alpha)\left(1-\lambda_{1}\right)+(1-\alpha)^{2} \sigma_{0}^{2} .
\end{aligned}
$$

This completes the proof.

Theorem 2.7. For $0<\alpha<1$, the vertex probability distribution $f_{k}$ of an $\alpha$-lazy random walk on an Eulerian directed graph $D$ at time $k$ converges to the stationary distribution $\pi$ in probability. In particular, we have

$$
\left\|\left(f_{k}-\pi\right) T^{-1 / 2}\right\| \leq \sigma_{\alpha}^{k}\left\|\left(f_{0}-\pi\right) T^{-1 / 2}\right\| .
$$

Here $f_{0}$ is the initial vertex probability distribution. 
The proof is omitted, since it is very similar to the proof of Theorem 2.1. Notice that when $0<\alpha<1$, we have $\sigma_{\alpha}<1$ by Lemma 2.6. The $\alpha$-lazy random walk converges to the stationary distribution exponentially fast.

For two vertex subsets $X$ and $Y$ of $V(D)$, let $E(X, Y)$ be the number of directed edges from $X$ to $Y$, i.e., $E(X, Y)=\{(u, v): u \in X$ and $v \in Y\}$. We have the following theorem on edge expansions in Eulerian directed graphs.

Theorem 2.8. If $X$ and $Y$ are two subsets of the vertex set $V$ of an Eulerian directed graph $D$, then we have

$$
|| E(X, Y)\left|-\frac{\operatorname{vol}(X) \operatorname{vol}(Y)}{\operatorname{vol}(D)}\right| \leq \sigma_{0} \frac{\sqrt{\operatorname{vol}(X) \operatorname{vol}(Y) \operatorname{vol}(\bar{X}) \operatorname{vol}(\bar{Y})}}{\operatorname{vol}(D)} .
$$

Proof. Let $\mathbf{1}_{X}$ be the indicator variable of $X$, i.e., $\mathbf{1}_{X}(u)=1$ if $u \in X$ and $\mathbf{1}_{X}(u)=0$ otherwise. We define $\mathbf{1}_{Y}$ similarly. Assume $\mathbf{1}_{X} T^{1 / 2}=a_{0} \phi_{0}+a_{1} \phi_{1}$ and $\mathbf{1}_{Y} T^{1 / 2}=b_{0} \phi_{0}+b_{1} \phi_{2}$, where $\phi_{1}, \phi_{2}$ are unit vectors in $\phi_{0}^{\perp}$. Since $\phi_{0}$ is a unit vector, we have

$$
a_{0}=\left\langle\mathbf{1}_{X} T^{1 / 2}, \phi_{0}\right\rangle=\frac{\operatorname{vol}(X)}{\sqrt{\operatorname{vol}(D)}}
$$

and

$$
a_{0}^{2}+a_{1}^{2}=\left\langle\mathbf{1}_{X} T^{1 / 2}, \mathbf{1}_{X} T^{1 / 2}\right\rangle=\operatorname{vol}(X)
$$

Thus

$$
a_{1}=\sqrt{\operatorname{vol}(X) \operatorname{vol}(\bar{X}) / \operatorname{vol}(D)}
$$

Similarly, we get

$$
b_{0}=\frac{\operatorname{vol}(Y)}{\sqrt{\operatorname{vol}(D)}} \quad \text { and } \quad b_{1}=\sqrt{\operatorname{vol}(Y) \operatorname{vol}(\bar{Y}) / \operatorname{vol}(D)} .
$$

It follows that

$$
\begin{aligned}
& || E(X, Y)\left|-\frac{\operatorname{vol}(X) \operatorname{vol}(Y)}{\operatorname{vol}(D)}\right| \\
& =\left|\mathbf{1}_{X} T^{1 / 2}\left(L_{0}-\phi_{0}^{\prime} \phi_{0}\right)\left(\mathbf{1}_{Y} T^{1 / 2}\right)^{\prime}\right| \\
& =\left|\left(a_{0} \phi_{0}+a_{1} \phi_{1}\right)\left(L_{0}-\phi_{0}^{\prime} \phi_{0}\right)\left(b_{0} \phi_{0}+b_{1} \phi_{2}\right)^{\prime}\right|=\left|a_{1} b_{1} \phi_{1} L_{0} \phi_{2}^{\prime}\right| \\
& \leq\left|a_{1} b_{1}\right|\left\|\phi_{1}|||| L_{0} \phi_{2}^{\prime}\right\| \leq\left|a_{1} b_{1}\right| \sigma_{0}=\sigma_{0} \frac{\sqrt{\operatorname{vol}(X) \operatorname{vol}(Y) \operatorname{vol}(\bar{X}) \operatorname{vol}(\bar{Y})}}{\operatorname{vol}(D)} .
\end{aligned}
$$

The proof of the theorem is complete. 
If we use $\bar{\lambda}$ instead of $\sigma_{0}$, then we get a weaker theorem on edge expansions. The proof will be omitted, since it is very similar to the proof of Theorem 2.8.

Theorem 2.9. Let $D$ be an Eulerian directed graph. If $X$ and $Y$ are two subsets of $V(D)$, then we have

$$
\left|\frac{|E(X, Y)|+|E(Y, X)|}{2}-\frac{\operatorname{vol}(X) \operatorname{vol}(Y)}{\operatorname{vol}(D)}\right| \leq \bar{\lambda} \cdot \frac{\sqrt{\operatorname{vol}(X) \operatorname{vol}(Y) \operatorname{vol}(\bar{X}) \operatorname{vol}(\bar{Y})}}{\operatorname{vol}(D)} .
$$

For $X, Y \subseteq V(D)$, let $d(X, Y)=\min \{d(u, v): u \in X$ and $v \in Y\}$. We have the following upper bound on $d(X, Y)$.

Theorem 2.10. Suppose that $D$ is a connected Eulerian directed graph. For $X, Y \subseteq$ $V(D)$ such that $d(X, Y) \geq 2$ and $0 \leq \alpha<1$, we have

$$
d(X, Y) \leq\left\lfloor\log \sqrt{\frac{\operatorname{vol}(\bar{X}) \operatorname{vol}(\bar{Y})}{\operatorname{vol}(X) \operatorname{vol}(Y)}} / \log \sigma_{\alpha}\right\rfloor+1
$$

In particular, for $0 \leq \alpha<1$, the diameter of $D$ satisfies

$$
\operatorname{diam}(D) \leq\left\lceil\frac{\log (\operatorname{vol}(D) / \delta)}{\log \sigma_{\alpha}}\right\rceil
$$

where $\delta=\min \left\{d_{x}: x \in V\right\}$.

Remark 2.II. From Lemma 2.6, we have

$$
\sigma_{\alpha}^{2} \leq \alpha^{2}+2 \alpha(1-\alpha) \lambda_{1}+(1-\alpha)^{2} \sigma_{0}^{2} .
$$

We can choose $\alpha$ to minimize $\sigma_{\alpha}$. If $\lambda_{1} \leq 1-\sigma_{0}^{2}$, then we choose $\alpha=0$ and get $\sigma_{\alpha}=\sigma_{0}$; if $\lambda_{1}>1-\sigma_{0}^{2}$, then we choose

$$
\alpha=\frac{\lambda_{1}+\sigma_{0}^{2}-1}{2 \lambda_{1}+\sigma_{0}^{2}-1}
$$

and get

$$
\sigma_{\alpha}^{2} \leq 1-\frac{\lambda_{1}^{2}}{2 \lambda_{1}+\sigma_{0}^{2}-1} .
$$

Combining the two cases, we have

$$
\min _{0 \leq \alpha<1}\left\{\sigma_{\alpha}\right\} \leq \begin{cases}\sigma_{0} & \text { if } \lambda_{1} \leq 1-\sigma_{0}^{2}, \\ \sqrt{1-\frac{\lambda_{1}^{2}}{2 \lambda_{1}+\sigma_{0}^{2}-1}} & \text { otherwise. }\end{cases}
$$


It is easy to check that

$$
\min _{0 \leq \alpha<1}\left\{\sigma_{\alpha}\right\} \leq \sqrt{1-\frac{\lambda_{1}}{2}}
$$

Here the inequality is strict if $\sigma_{0}<1$. We have

$$
d(X, Y) \leq\left\lfloor\log \frac{\operatorname{vol}(\bar{X}) \operatorname{vol}(\bar{Y})}{\operatorname{vol}(X) \operatorname{vol}(Y)} / \log \frac{2}{2-\lambda_{1}}\right\rfloor+1
$$

Theorem 2.10 is stronger than Theorem 2.5 in general.

Proof. Similar to what we did in the proof of Theorem 2.8, let $\mathbf{1}_{X}$ and $\mathbf{1}_{Y}$ be the indicator functions of $X$ and $Y$, respectively. We have

$$
\mathbf{1}_{X} T^{1 / 2}=a_{0} \phi_{0}+a_{1} \phi_{1} \quad \text { and } \quad \mathbf{1}_{Y} T^{1 / 2}=b_{0} \phi_{0}+b_{1} \phi_{2},
$$

where $\phi_{1}, \phi_{2}$ are unit vectors in $\phi_{0}^{\perp}$, and $a_{0}, b_{0}, a_{1}, b_{1}$ are given by (2.5) through (2.8).

Let

$$
k=\left\lfloor\log \sqrt{\frac{\operatorname{vol}(\bar{X}) \operatorname{vol}(\bar{Y})}{\operatorname{vol}(X) \operatorname{vol}(Y)}} / \log \sigma_{\alpha}\right\rfloor+1
$$

We have

$$
\left(\mathbf{1}_{X} T^{1 / 2}\right) L_{\alpha}^{k}\left(\mathbf{1}_{Y} T^{1 / 2}\right)^{\prime} \geq a_{0} b_{0}+\sigma_{\alpha}^{k} a_{1} b_{1}>0 .
$$

Thus there is a directed path starting from some vertex in $X$ and ending at some vertex in $Y$, that is, $d(X, Y) \leq k$.

For the diameter result, we choose $X=\{x\}$ and $Y=\{y\}$. Note that $\operatorname{vol}(X)=$ $d_{x} \geq \delta, \operatorname{vol}(Y)=d_{y} \geq \delta, \operatorname{vol}(\bar{X})<\operatorname{vol}(G)$, and $\operatorname{vol}(\bar{y})<\operatorname{vol}(G)$. The result follows.

\section{Definition of the sth Laplacian}

Let $H$ be an $r$-uniform hypergraph (or an $r$-graph for short) with vertex set $V(H)$ (or $V$ for short) and edge set $E(H)$. We assume that $|V(H)|=n$ and $E(H) \subseteq\left(\begin{array}{l}V \\ r\end{array}\right)$. For a vertex subset $S$ such that $|S|<r$, the neighborhood $\Gamma(S)$ is $\{T \mid S \cap T=\varnothing$ and $S \cup T$ is an edge in $H\}$. Let the degree $d_{S}$ of $S$ in $H$ be the number of edges containing $S$, i.e., $d_{S}=|\Gamma(S)|$. For $1 \leq s \leq r-1$, an $s$-walk of length $k$ is a sequence of vertices

$$
v_{1}, v_{2}, \ldots, v_{j}, \ldots, v_{(r-s)(k-1)+r}
$$




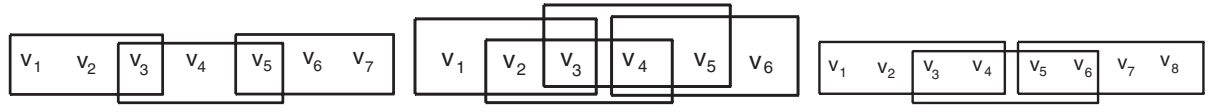

Figure I. Three examples of an $s$-walk in a hypergraph: a 1-walk in a 3-graph (left), a 2 -walk in a 3 -graph (center), and a 2-walk in a 4-graph right).

together with a sequence of edges $F_{1}, F_{2}, \ldots, F_{k}$ such that

$$
F_{i}=\left\{v_{(r-s)(i-1)+1}, v_{(r-s)(i-1)+2}, \ldots, v_{(r-s)(i-1)+r}\right\}
$$

for $1 \leq i \leq k$. Some examples of $s$-walks are shown in Figure 1 .

For each $i$ in $\{0,1, \ldots, k\}$, the $i$ th stop $x_{i}$ of an $s$-walk is the ordered $s$-tuple $\left(v_{(r-s) i+1}, v_{(r-s) i+2}, \ldots, v_{(r-s) i+s}\right)$. The initial stop is $x_{0}$, and the terminal stop is $x_{k}$. An $s$-walk is an $s$-path if stops (as ordered $s$-tuples) are distinct. If $x_{0}=x_{k}$, then an $s$-walk is closed.

An $s$-cycle is a special closed $s$-walk such that $v_{1}, v_{2}, \ldots, v_{(r-s) k}$ are distinct and $v_{(r-s) k+j}=v_{j}$ for $1 \leq j \leq s$ (see Figure 2). An $s$-cycle is a loose cycle if $s \leq r / 2$ (particularly $s=1$ ); an $s$-cycle is a tight cycle if $s>r / 2$ (particularly $s=r-1)$. An $s$-cycle is Hamiltonian if it covers each vertex in $H$ exactly once. In the literature, Hamiltonian tight cycles were first studied in [Katona and Kierstead 99]. Hamiltonian $s$-cycles for a full range of $s$ were studied in [Rödl et al. 06, Rödl et al. 08, Keevash et al. 12, Kühn et al. 12, Kühn and Osthus 06, Hàn and Schacht 99]. Hamiltonian $s$-cycles in random $r$-uniform hypergraphs were studied in [Dudek and Frieze 11, Dudek and Frieze 12, Frieze 10].

For $1 \leq s \leq r-1$ and $x, y \in \mathrm{V}^{\underline{s}}$, the $s$-distance $d^{(s)}(x, y)$ is the minimum integer $k$ such that there exists an $s$-path of length $k$ starting from $x$ and ending at $y$. A hypergraph $H$ is $s$-connected if $d^{(s)}(x, y)$ is finite for every pair $(x, y)$. If $H$ is $s$-connected, then the $s$-diameter of $H$ is the maximum value of $d^{(s)}(x, y)$ for $x, y \in \mathrm{V}^{\underline{s}}$.
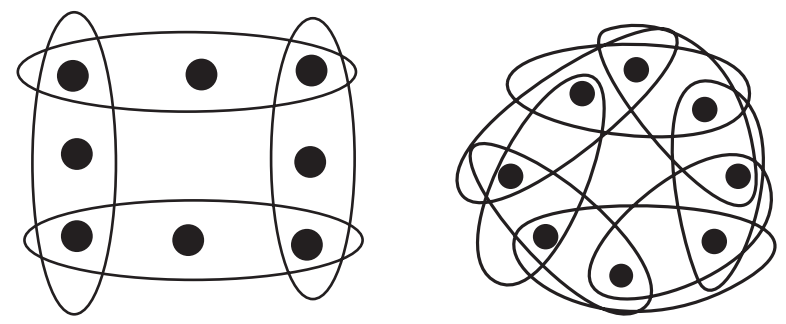

Figure 2. Examples of a loose cycle and a tight cycle in a 3-graph: a 1-cycle in a 3 -graph (left) and a 2-cycle in a 3 -graph (right). 
A random $s$-walk with initial stop $x_{0}$ is an $s$-walk generated as follows. Let $x_{0}$ be the sequence of visited vertices at the initial step. At each step, let $S$ be the set of the last $s$ vertices in the sequence of visited vertices. A random $(r-s)$-set $T$ is chosen from $\Gamma(S)$ uniformly; the vertices in $T$ are added into the sequence one by one in an arbitrary order.

For $0 \leq \alpha \leq 1$, an $\alpha$-lazy random $s$-walk is a modified random $s$-walk such that with probability $\alpha$, one can stay at the current stop; with probability $1-\alpha$, append $r-s$ vertices to the sequence as selected in a random $s$-walk.

For $x \in \mathrm{V}^{\underline{s}}$, let $[x]$ be the $s$-set consisting of the coordinates of $x$.

\section{I. Case I $\leq s \leq r / 2$}

For $1 \leq s \leq r / 2$, we define a weighted undirected graph $G^{(s)}$ over the vertex set $\mathrm{V}^{\underline{s}}$ as follows. Let the weight $w(x, y)$ be $|\{F \in E(H):[x] \sqcup[y] \subseteq F\}|$. Here $[x] \sqcup[y]$ is the disjoint union of $[x]$ and $[y]$. In particular, if $[x] \cap[y] \neq \varnothing$, then $w(x, y)=0$.

For $x \in \mathrm{V}^{\underline{s}}$, the degree of $x$ in $G^{(s)}$, denoted by $d_{x}^{(s)}$, is given by

$$
d_{x}^{(s)}=\sum_{y} w(x, y)=d_{[x]}\left(\begin{array}{c}
r-s \\
s
\end{array}\right) s !
$$

Here $d_{[x]}$ means the degree of the set $[x]$ in the hypergraph $H$. When we restrict an $s$-walk on $H$ to its stops, we get a walk on $G^{(s)}$. This restriction preserves the length of the walk. Therefore, the $s$-distance $d^{(s)}(x, y)$ in $H$ is simply the graph distance between $x$ and $y$ in $G^{(s)}$; the $s$-diameter of $H$ is simply the diameter of the graph $G^{(s)}$.

A random $s$-walk on $H$ is essentially a random walk on $G^{(s)}$. It can be constructed from a random walk on $G^{(s)}$ by inserting $r-2 s$ additional random vertices $T_{i}$ between two consecutive stops $x_{i}$ and $x_{i+1}$ at time $i$, where $T_{i}$ is chosen uniformly from $\Gamma\left(\left[x_{i}\right] \cup\left[x_{i+1}\right]\right)$ and the vertices $T_{i}$ are inserted between $x_{i}$ and $x_{i+1}$ in an arbitrary order.

Therefore, we define the sth Laplacian $\mathcal{L}^{(s)}$ of $H$ to be the Laplacian of the weighted undirected graph $G^{(s)}$.

The eigenvalues of $\mathcal{L}^{(s)}$ are listed as $\lambda_{0}^{(s)}, \lambda_{1}^{(s)}, \ldots, \lambda_{\left(\begin{array}{c}n \\ s\end{array}\right) s !-1}^{(s)}$ in nondecreasing order. Let $\lambda_{\max }^{(s)}=\lambda_{\left(\begin{array}{c}n \\ s\end{array}\right) s !-1}^{(s)}$ and $\bar{\lambda}^{(s)}=\max \left\{\left|1-\lambda_{1}^{(s)}\right|,\left|1-\lambda_{\max }^{(s)}\right|\right\}$. For some hypergraphs, the numerical values of $\lambda_{1}^{(s)}$ and $\lambda_{\max }^{(s)}$ are shown in Table 1. 


\begin{tabular}{c|cccccccc}
$H$ & $\lambda_{1}^{(4)}$ & $\lambda_{1}^{(3)}$ & $\lambda_{1}^{(2)}$ & $\lambda_{1}^{(1)}$ & $\lambda_{\max }^{(1)}$ & $\lambda_{\max }^{(2)}$ & $\lambda_{\max }^{(3)}$ & $\lambda_{\max }^{(4)}$ \\
\hline$K_{6}^{3}$ & & & $3 / 4$ & $6 / 5$ & $6 / 5$ & $3 / 2$ & & \\
$K_{7}^{3}$ & & & $7 / 10$ & $7 / 6$ & $7 / 6$ & $3 / 2$ & & \\
$K_{6}^{4}$ & & $1 / 3$ & $5 / 6$ & $6 / 5$ & $6 / 5$ & $3 / 2$ & 1.76759 & \\
$K_{7}^{4}$ & & $3 / 8$ & $9 / 10$ & $7 / 6$ & $7 / 6$ & $7 / 5$ & $7 / 4$ & \\
$K_{6}^{5}$ & 0.1464 & $1 / 2$ & $5 / 6$ & $6 / 5$ & $6 / 5$ & $3 / 2$ & $3 / 2$ & 1.809 \\
$K_{7}^{5}$ & 0.1977 & $5 / 8$ & $9 / 10$ & $7 / 6$ & $7 / 6$ & $7 / 5$ & $3 / 2$ & 1.809
\end{tabular}

Table I. The values of $\lambda_{1}^{(s)}$ and $\lambda_{\max }^{(s)}$ of some complete hypergraphs $K_{n}^{r}$.

\subsection{The case $r / 2<s \leq r-1$}

For $r / 2<s \leq r-1$, we define a directed graph $D^{(s)}$ over the vertex set $\mathrm{V}^{\underline{s}}$ as follows. For $x, y \in \mathrm{V}^{\underline{s}}$ such that $x=\left(x_{1}, \ldots, x_{s}\right)$ and $y=\left(y_{1}, \ldots, y_{s}\right)$, let $(x, y)$ be a directed edge if $x_{r-s+j}=y_{j}$ for $1 \leq j \leq 2 s-r$ and $[x] \cup[y]$ is an edge of $H$.

For $x \in \mathrm{V}^{\underline{s}}$, the out-degree $d_{x}^{+}$in $D^{(s)}$ and the in-degree $d_{x}^{-}$in $D^{(s)}$ satisfy

$$
d_{x}^{+}=d_{[x]}(r-s) !=d_{x}^{-} .
$$

Thus $D^{(s)}$ is an Eulerian directed graph. We write $d_{x}^{(s)}$ for both $d_{x}^{+}$and $d_{x}^{-}$. Now $D^{(s)}$ is strongly connected if and only if it is weakly connected.

Note that an $s$-walk on $H$ can be naturally viewed as a walk on $D^{(s)}$ and vice versa. Thus the $s$-distance $d^{(s)}(x, y)$ in $H$ is exactly the directed distance from $x$ to $y$ in $G^{(s)}$; the $s$-diameter of $H$ is the diameter of $D^{(s)}$. A random $s$-walk on $H$ is in one-to-one correspondence with a random walk on $D^{(s)}$.

For $r / 2<s \leq r-1$, we define the sth Laplacian $\mathcal{L}^{(s)}$ as the Laplacian of the Eulerian directed graph $D^{(s)}$ (see Section 2).

The eigenvalues of $\mathcal{L}^{(s)}$ are listed as $\lambda_{0}^{(s)}, \lambda_{1}^{(s)}, \ldots, \lambda_{\left(\begin{array}{l}n \\ s\end{array}\right) s !-1}^{(s)}$ in nondecreasing

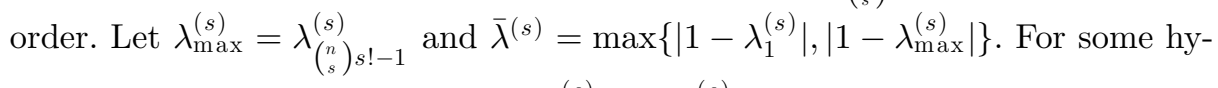
pergraphs, the numerical values of $\lambda_{1}^{(s)}$ and $\lambda_{\max }^{(s)}$ are shown in Table 1.

\subsection{Examples}

Let $K_{n}^{r}$ be the complete $r$-uniform hypergraph on $n$ vertices. Here we compute the values of $\lambda_{1}^{(s)}$ and $\lambda_{\max }^{(s)}$ for some $K_{n}^{r}$ (see Table 1 ).

From Table 1, we observe that $\lambda_{1}^{(s)}=\lambda_{\max }^{(s)}$ for some complete hypergraphs. In fact, this is true for every complete hypergraph $K_{n}^{r}$. We have the following property. 
Property 3.I. For an r-uniform hypergraph $H$ and an integer s such that $1 \leq s \leq r / 2$, we have $\lambda_{1}^{(s)}(H)=\lambda_{\max }^{(s)}(H)$ if and only if $s=1$ and $H$ is a 2-design.

Proof. In one direction, suppose $s=1$ and $H$ is a 2-design. For each pair of vertices, the number of edges containing the pair is a constant. Thus $G^{(s)}$ is a complete weighted graph. The Laplacian of any complete weighted graph is the same as the Laplacian of the complete graph $K_{n}$. Thus, $\lambda_{1}^{(s)}(H)=\lambda_{\max }^{(s)}(H)$.

In the other direction, suppose $\lambda_{1}^{(s)}(H)=\lambda_{\max }^{(s)}(H)=\lambda$. We have

$$
\mathcal{L}^{(s)}=\lambda I-\lambda \phi_{0}^{*} \phi_{0},
$$

where $\phi_{0}$ is the unit eigenvector corresponding to the trivial eigenvalue 0 . Taking the trace of both sides, we get

$$
\left(\begin{array}{l}
n \\
s
\end{array}\right) s !=\lambda\left(\begin{array}{l}
n \\
s
\end{array}\right) s !-\lambda
$$

Solving for $\lambda$, we get

$$
\lambda=1+\frac{1}{\left(\begin{array}{l}
n \\
s
\end{array}\right) s !-1} .
$$

Write $\mathcal{L}^{(s)}=I-T^{-1 / 2} A T^{-1 / 2}$, where $A$ is the weight matrix of $G^{(s)}$ and $T$ is the diagonal matrix of $d_{x}^{(s)}$ in $G^{(s)}$. From (3.2), we have

$$
T^{-1 / 2} A T^{-1 / 2}=-\frac{1}{\left(\begin{array}{l}
n \\
s
\end{array}\right) s !-1} I+\frac{\left(\begin{array}{l}
n \\
s
\end{array}\right) s !}{\left(\begin{array}{l}
n \\
s
\end{array}\right) s !-1} \phi_{0}^{*} \phi_{0} .
$$

If $s \geq 2$, then some off-diagonal entries of $A$ are zero by the definition of $G^{(s)}$. However, all off-diagonal entries of the right-hand-side matrix are nonzero, a contradiction. We must have $s=1$. In this case, we have

$$
\phi_{0}=\frac{1}{\operatorname{vol}\left(G^{(1)}\right)}\left(\sqrt{d_{1}}, \ldots, \sqrt{d_{n}}\right)^{*} .
$$

By comparing the diagonal entries of (3.3), we get $d_{1}=d_{2}=\cdots=d_{n}$. Thus $H$ is a 2-design.

\section{Properties of Laplacians}

In this section, we prove some properties of the Laplacians for hypergraphs.

Lemma 4.I. For $1 \leq s \leq r / 2$, we have the following properties: 
1. The sth Laplacian has $\left(\begin{array}{l}n \\ s\end{array}\right)$ s! eigenvalues, and all of them are in $[0,2]$.

2. The number of 0 eigenvalues is the number of connected components in $G^{(s)}$.

3. The Laplacian $\mathcal{L}^{(s)}$ has an eigenvalue 2 if and only if $r=2 s$ and $G^{(s)}$ has a bipartite component.

Proof. We have the following facts (see [Chung 97, Chapter 1]) for a weighted graph $G$ : all Laplacian eigenvalues of $G$ are in $[0,2]$; the number of 0 eigenvalues is the number of connected components in $G$; $G$ has an eigenvalue 2 if and only if $G$ has a bipartite component.

Items 1 and 2 follow from the facts of the Laplacian of $G^{(s)}$. We thus need to prove only item 3 . If $\mathcal{L}^{(s)}$ has an eigenvalue 2 , then $G^{(s)}$ has a bipartite component $T$. We want to show that $r=2 s$. Suppose $r \geq 2 s+1$. Let $\left\{v_{0}, v_{1}, \ldots, v_{r-1}\right\}$ be an edge in $T$. For $0 \leq i \leq 2 s+1$ and $0 \leq j \leq s-1$, let $g(i, j)=i s+j \bmod (2 s+$ $1)$ and $x_{i}=\left(v_{g(i, 0)}, \ldots, v_{g(i, s-1)}\right)$. Observe that $\left[x_{i}\right] \cap\left[x_{i+1}\right]=\varnothing$ for all $0 \leq i \leq$ $2 s$ and $x_{2 s+1}=x_{0}$. Thus the sequence $x_{0}, x_{1}, \ldots, x_{2 s}$ forms an odd cycle in $G^{(s)}$, a contradiction.

The following lemma compares $\lambda_{1}^{(s)}$ and $\lambda_{\max }^{(s)}$ for different $s$.

Lemma 4.2. Suppose that $H$ is an $r$-uniform hypergraph. We have

$$
\begin{gathered}
\lambda_{1}^{(1)} \geq \lambda_{1}^{(2)} \geq \cdots \geq \lambda_{1}^{(\lfloor r / 2\rfloor)} ; \\
\lambda_{\max }^{(1)} \leq \lambda_{\max }^{(2)} \leq \cdots \leq \lambda_{\max }^{(\lfloor r / 2\rfloor)} .
\end{gathered}
$$

Remark 4.3. We do not know whether similar inequalities hold for $s>r / 2$.

Proof. Let $T_{s}$ be the diagonal matrix of degrees in $G^{(s)}$, and let $R^{(s)}(f)$ be the Rayleigh quotient of $\mathcal{L}^{(s)}$. It suffices to show that $\lambda_{1}^{(s)} \leq \lambda_{1}^{(s-1)}$ for $2 \leq s \leq r / 2$. Recall that $\lambda_{1}^{(s)}$ can be defined via the Rayleigh quotient; see (2.1). Pick a function $f: V^{(s-1)} \rightarrow R$ such that $\left\langle f, T_{s-1} \mathbf{1}\right\rangle=0$ and $\lambda_{1}^{(s-1)}=R^{(s-1)}(f)$. We define $g: \mathrm{V} \underline{s} \rightarrow R$ as follows:

$$
g(x)=f\left(x^{\prime}\right)
$$

where $x^{\prime}$ is an $(s-1)$-tuple consisting of the first $(s-1)$ coordinates of $x$ with the same order in $x$. Applying (3.1), we get

$$
\left\langle g, T_{s} \mathbf{1}\right\rangle=\sum_{x \in \mathrm{V}^{\underline{s}}} d_{x}^{(s)} g(x)=\sum_{x \in \mathrm{V}^{\underline{s}}} g(x) d_{[x]}\left(\begin{array}{c}
r-s \\
s
\end{array}\right) s ! .
$$


We have

$$
\begin{aligned}
& \sum_{x} g(x) d_{[x]}=\sum_{x} \sum_{F:[x] \subseteq F} g(x) \\
& =\sum_{x^{\prime}} \sum_{F:\left[x^{\prime}\right] \subseteq F}(r-s+1) f\left(x^{\prime}\right)=\sum_{x^{\prime}} d_{\left[x^{\prime}\right]}(r-s+1) f\left(x^{\prime}\right) \\
& =\frac{r-s+1}{\left(\begin{array}{c}
r-s+1 \\
s-1
\end{array}\right)(s-1) !} \sum_{x^{\prime}} f\left(x^{\prime}\right) d_{x^{\prime}}^{(s-1)}=0 .
\end{aligned}
$$

Here the second-to-last equality follows from (3.1), and the last one follows from the choice of $f$. Therefore,

$$
\sum_{x} g(x) d_{x}^{(s)}=(r-s+2)(r-s+1) \sum_{x^{\prime}} f\left(x^{\prime}\right) d_{x^{\prime}}^{(s-1)} .
$$

Thus $\left\langle g, T_{s} \mathbf{1}\right\rangle=0$. Similarly, we have

$$
\sum_{x} g(x)^{2} d_{x}^{(s)}=(r-s+2)(r-s+1) \sum_{x^{\prime}} f\left(x^{\prime}\right)^{2} d_{x^{\prime}}^{(s-1)} .
$$

Putting these together, we obtain

$$
\sum_{x} g(x)^{2} d_{x}^{(s)}=(r-s+2)(r-s+1) \sum_{x^{\prime}} f\left(x^{\prime}\right)^{2} d_{x^{\prime}}^{(s-1)} .
$$

By a similar counting method, we have

$$
\begin{aligned}
& \sum_{x \sim y}(g(x)-g(y))^{2} w(x, y)=\sum_{x \sim y} \sum_{F:[x] \sqcup[y] \subseteq F}(g(x)-g(y))^{2} \\
& =\sum_{x^{\prime} \sim y^{\prime} F:\left[x^{\prime}\right] \sqcup\left[y^{\prime}\right] \subseteq F}(r-s+1)(r-s+2)\left(f\left(x^{\prime}\right)-f\left(y^{\prime}\right)\right)^{2} \\
& =(r-s+1)(r-s+2) \sum_{x^{\prime} \sim y^{\prime}}\left(f\left(x^{\prime}\right)-f\left(y^{\prime}\right)\right)^{2} w\left(x^{\prime}, y^{\prime}\right) .
\end{aligned}
$$

Thus $R^{(s)}(g)=R^{(s-1)}(f)=\lambda_{1}^{(s-1)}$ by the choice of $f$. Since $\lambda_{1}^{(s)}$ is the infimum over all $g$, we get $\lambda_{1}^{(s)} \leq \lambda_{1}^{(s-1)}$.

The inequality (4.2) can be proved similarly. Since $\lambda_{\max }^{(s)}$ is the supremum of the Rayleigh quotient, the direction of the inequalities is reversed.

Lemma 4.4. For $r / 2<s \leq r-1$, we have the following facts:

1. The sth Laplacian has $\left(\begin{array}{l}n \\ s\end{array}\right)$ s! eigenvalues, and all of them are in $[0,2]$.

2. The number of 0 eigenvalues is the number of strongly connected components in $D^{(s)}$. 
3. If 2 is an eigenvalue of $\mathcal{L}^{(s)}$, then one of the s-connected components of $H$ is bipartite.

The proof is trivial and will be omitted.

\section{Applications}

In this section, we show some applications of Laplacians $\mathcal{L}^{(s)}$ of hypergraphs.

\section{I. Random $s$-Walks on Hypergraphs}

For $0 \leq \alpha<1$ and $1 \leq s \leq r / 2$, after restricting an $\alpha$-lazy random $s$-walk on a hypergraph $H$ to its stops (see Section 3), we get an $\alpha$-lazy random walk on the corresponding weighted graph $G^{(s)}$. Let $\pi(x)=d_{x} / \operatorname{vol}\left(\mathrm{V}^{\underline{s}}\right)$ for all $x \in \mathrm{V}^{\underline{s}}$, where $d_{x}$ is the degree of $x$ in $G^{(s)}$ and $\operatorname{vol}\left(\mathrm{V}^{\underline{s}}\right)$ is the volume of $G^{(s)}$. Applying Theorem 2.1, we have the following theorem.

Theorem 5.I. For $1 \leq s \leq r / 2$, suppose that $H$ is an $s$-connected $r$-uniform hypergraph and $\lambda_{1}^{(s)}$ is the first nontrivial eigenvalue of the sth Laplacian of $H$, while $\lambda_{\max }^{(s)}$ is the last. For $0 \leq \alpha<1$, the joint distribution $f_{k}$ at the kth stop of an $\alpha$-lazy random walk at time $k$ converges to the stationary distribution $\pi$ in probability. In particular, we have

$$
\left\|\left(f_{k}-\pi\right) T^{-1 / 2}\right\| \leq\left(\bar{\lambda}_{\alpha}^{(s)}\right)^{k}\left\|\left(f_{0}-\pi\right) T^{-1 / 2}\right\|,
$$

where

$$
\bar{\lambda}_{\alpha}^{(s)}=\max \left\{\left|1-(1-\alpha) \lambda_{1}^{(s)}\right|,\left|(1-\alpha) \lambda_{\max }^{(s)}-1\right|\right\},
$$

and $f_{0}$ is the probability distribution at the initial stop.

For $0<\alpha<1$ and $r / 2<s \leq r-1$, when restricting an $\alpha$-lazy random $s$-walk on a hypergraph $H$ to its stops (see Section 2), we get an $\alpha$-lazy random walk on the corresponding directed graph $D^{(s)}$. Let $\pi(x)=d_{x} / \operatorname{vol}\left(\mathrm{V}^{\underline{s}}\right)$ for all $x \in \mathrm{V}^{\underline{s}}$, where $d_{x}$ is the degree of $x$ in $D^{(s)}$ and $\operatorname{vol}\left(\mathrm{V}^{\underline{s}}\right)$ is the volume of $D^{(s)}$. Applying Theorem 2.7, we have the following theorem.

Theorem 5.2. For $r / 2<s \leq r-1$, suppose that $H$ is an $s$-connected $r$-uniform hypergraph and $\lambda_{1}^{(s)}$ is the first nontrivial eigenvalue of the sth Laplacian of $H$. For $0<\alpha<1$, the joint distribution $f_{k}$ at the kth stop of an $\alpha$-lazy random walk 
at time $k$ converges to the stationary distribution $\pi$ in probability. In particular, we have

$$
\left\|\left(f_{k}-\pi\right) T^{-1 / 2}\right\| \leq\left(\sigma_{\alpha}^{(s)}\right)^{k}\left\|\left(f_{0}-\pi\right) T^{-1 / 2}\right\|,
$$

where $\sigma_{\alpha}^{(s)} \leq \sqrt{1-2 \alpha(1-\alpha) \lambda_{1}^{(s)}}$, and $f_{0}$ is the probability distribution at the initial stop.

Remark 5.3. The reason that we require $0<\alpha<1$ in the case $r / 2<s \leq r-1$ is that $\sigma_{0}\left(D^{(s)}\right)=1$ for $r / 2<s \leq r-1$.

\subsection{The $s$-Distances and $s$-Diameters in Hypergraphs}

Let $H$ be an $r$-uniform hypergraph. For $1 \leq s \leq r-1$ and $x, y \in \mathrm{V} \underline{s}$, the $s$ distance $d^{(s)}(x, y)$ is the minimum integer $k$ such that there is an $s$-path of length $k$ starting at $x$ and ending at $y$. For $X, Y \subseteq \mathrm{V}^{s}$, let

$$
d^{(s)}(X, Y)=\min \left\{d^{(s)}(x, y) \mid x \in X, y \in Y\right\} .
$$

If $H$ is $s$-connected, then the $s$-diameter $\operatorname{diam}^{(s)}(H)$ satisfies

$$
\operatorname{diam}^{(s)}(H)=\max _{x, y \in \mathrm{V}^{\underline{s}}}\left\{d^{(s)}(x, y)\right\} .
$$

For $1 \leq s \leq r / 2$, the $s$-distances in $H$ and the $s$-diameter of $H$ are simply the respective graph distances in $G^{(s)}$ and diameter of $G^{(s)}$. Applying Theorem 2.2 and Corollary 2.3, we have the following theorems.

Theorem 5.4. Suppose $H$ is an r-uniform hypergraph. For integer s such that $1 \leq$ $s \leq r / 2$, let $\lambda_{1}^{(s)}$ be the first nontrivial eigenvalue of the sth Laplacian of $H$, and $\lambda_{\max }^{(s)}$ the last. Suppose $\lambda_{\max }^{(s)}>\lambda_{1}^{(s)}>0$. For $X, Y \subseteq \mathrm{V}^{s}$, if $d^{(s)}(X, Y) \geq 2$, then we have

$$
d^{(s)}(X, Y) \leq\left\lceil\log \sqrt{\frac{\operatorname{vol}(\bar{X}) \operatorname{vol}(\bar{Y})}{\operatorname{vol}(X) \operatorname{vol}(Y)}} / \log \frac{\lambda_{\max }^{(s)}+\lambda_{1}^{(s)}}{\lambda_{\max }^{(s)}-\lambda_{1}^{(s)}}\right\rceil .
$$

Here $\operatorname{vol}(*)$ are volumes in $G^{(s)}$.

Remark 5.5. We know that $\lambda_{1}^{(s)}>0$ if and only if $H$ is $s$-connected. The condition $\lambda_{\max }^{(s)}>\lambda_{1}^{(s)}$ holds unless $s=1$ and every pair of vertices is covered by edges evenly (i.e., $H$ is a 2 -design).

Theorem 5.6. Suppose $H$ is an r-uniform hypergraph. For integer s such that $1 \leq$ $s \leq r / 2$, let $\lambda_{1}^{(s)}$ be the first nontrivial eigenvalue of the sth Laplacian of $H$, and 
let $\lambda_{\max }^{(s)}$ be the last. If $\lambda_{\max }^{(s)}>\lambda_{1}^{(s)}>0$, then the s-diameter of an r-uniform hypergraph $H$ satisfies

$$
\operatorname{diam}^{(s)}(H) \leq\left\lceil\log \frac{\operatorname{vol}\left(\mathrm{V}^{\underline{s}}\right)}{\delta^{(s)}} / \log \frac{\lambda_{\max }^{(s)}+\lambda_{1}^{(s)}}{\lambda_{\max }^{(s)}-\lambda_{1}^{(s)}}\right\rceil .
$$

Here

$$
\operatorname{vol}\left(\mathrm{V}^{\underline{s}}\right)=\sum_{x \in \mathrm{V}^{\underline{s}}} d_{x}=|E(H)| \frac{r !}{(r-2 s) !},
$$

and $\delta^{(s)}$ is the minimum degree in $G^{(s)}$.

When $r / 2<s \leq r-1$, the $s$-distances in $H$ and the $s$-diameter of $H$ are respectively the directed distance in $D^{(s)}$ and the diameter of $D^{(s)}$. Applying Theorem 2.10 and Remark 2.11, we have the following theorems.

Theorem 5.7. Let $H$ be an $r$-uniform hypergraph. For $r / 2<s \leq r-1$ and $X, Y \subseteq$ $\mathrm{V}^{s}$, if $H$ is s-connected, then we have

$$
d^{(s)}(X, Y) \leq\left\lfloor\log \frac{\operatorname{vol}(\bar{X}) \operatorname{vol}(\bar{Y})}{\operatorname{vol}(X) \operatorname{vol}(Y)} / \log \frac{2}{2-\lambda_{1}^{(s)}}\right\rfloor+1 .
$$

Here $\lambda_{1}^{(s)}$ is the first nontrivial eigenvalue of the Laplacian of $D^{(s)}$, and $\operatorname{vol}(*)$ are volumes in $D^{(s)}$.

Theorem 5.8. For $r / 2<s \leq r-1$, suppose that an $r$-uniform hypergraph $H$ is $s$ connected. Let $\lambda_{1}^{(s)}$ be the smallest nonzero eigenvalue of the Laplacian of $D^{(s)}$. The s-diameter of $H$ satisfies

$$
\operatorname{diam}^{(s)}(H) \leq\left\lceil 2 \log \frac{\operatorname{vol}\left(\mathrm{V}^{\underline{s}}\right)}{\delta^{(s)}} / \log \frac{2}{2-\lambda_{1}^{(s)}}\right\rceil .
$$

Here $\operatorname{vol}\left(\mathrm{V}^{\underline{s}}\right)=\sum_{x \in \mathrm{V} \underline{s}} d_{x}=|E(H)| r$ ! and $\delta^{(s)}$ is the minimum degree in $D^{(s)}$.

\subsection{Edge Expansions in Hypergraphs}

In this subsection, we prove some results on edge expansions in hypergraphs. Note that there has been some attempt to generalize the edge discrepancy theorem from graphs to hypergraphs [Butler unpubl.]. 
Let $H$ be an $r$-uniform hypergraph. For $S \subseteq\left(\begin{array}{c}V \\ s\end{array}\right)$, we recall that the volume of $S$ satisfies

$$
\operatorname{vol}(S)=\sum_{x \in S} d_{x}
$$

Here $d_{x}$ is the degree of the set $x$ in $H$. In particular, we have

$$
\operatorname{vol}\left(\left(\begin{array}{l}
V \\
s
\end{array}\right)\right)=|E(H)|\left(\begin{array}{l}
r \\
s
\end{array}\right) .
$$

The density e $(S)$ of $S$ is $\operatorname{vol}(S) / \operatorname{vol}\left(\left(\begin{array}{c}V \\ s\end{array}\right)\right)$. Let $\bar{S}$ be the complement of $S$ in $\left(\begin{array}{c}V \\ s\end{array}\right)$. We have

$$
e(\bar{S})=1-e(S)
$$

For $1 \leq t \leq s \leq r-t, S \subseteq\left(\begin{array}{c}V \\ s\end{array}\right)$, and $T \subseteq\left(\begin{array}{c}V \\ t\end{array}\right)$, let

$$
E(S, T)=\{F \in E(H): \exists x \in S, \exists y \in T, x \cap y=\varnothing, \text { and } x \cup y \subseteq F\} .
$$

Note that $|E(S, T)|$ counts the number of edges contained in $x \sqcup y$ for some $x \in S$ and $y \in T$. In particular, we have

$$
\left|E\left(\left(\begin{array}{l}
V \\
s
\end{array}\right),\left(\begin{array}{l}
V \\
t
\end{array}\right)\right)\right|=|E(H)| \frac{r !}{s ! t !(r-s-t) !} .
$$

Theorem 5.9. For $1 \leq t \leq s \leq r / 2, S \subseteq\left(\begin{array}{c}V \\ s\end{array}\right)$, and $T \subseteq\left(\begin{array}{c}V \\ t\end{array}\right)$, let

$$
e(S, T)=\frac{|E(S, T)|}{\left|E\left(\left(\begin{array}{c}
V \\
s
\end{array}\right),\left(\begin{array}{c}
V \\
t
\end{array}\right)\right)\right|} .
$$

We have

$$
|e(S, T)-e(S) e(T)| \leq \bar{\lambda}^{(s)} \sqrt{e(S) e(T) e(\bar{S}) e(\bar{T})}
$$

Proof. Let $G^{(s)}$ be the weighed undirected graph defined in Section 3. Define $S^{\prime}$ and $T^{\prime}$ (sets of ordered $s$-tuples) as follows:

$$
S^{\prime}=\left\{x \in \mathrm{V}^{\underline{s}} \mid[x] \in S\right\}, \quad T^{\prime}=\left\{(y, z) \in \mathrm{V}^{\underline{s}} \mid[y] \in T\right\} .
$$

Let $\bar{S}^{\prime}$ and $\bar{T}^{\prime}$ be the respective complements of $S^{\prime}$ and $T^{\prime}$ in $\mathrm{V}^{\underline{s}}$. We make a convention that $\operatorname{vol}_{G^{(s)}}(*)$ denotes volumes in $G^{(s)}$, while $\operatorname{vol}(*)$ denotes volumes 
in $H$. We have

$$
\begin{aligned}
\operatorname{vol}_{G^{(s)}}\left(G^{(s)}\right) & =\operatorname{vol}\left(\left(\begin{array}{c}
V \\
s
\end{array}\right)\right) \frac{s !(r-s) !}{(r-2 s) !} \\
\operatorname{vol}_{G^{(s)}}\left(S^{\prime}\right) & =\operatorname{vol}(S) \frac{s !(r-s) !}{(r-2 s) !} \\
\operatorname{vol}_{G^{(s)}}\left(T^{\prime}\right) & =\operatorname{vol}(T) \frac{t !(r-t) !}{(r-2 s) !} \\
\operatorname{vol}_{G^{(s)}}\left(\bar{S}^{\prime}\right) & =\operatorname{vol}(\bar{S}) \frac{s !(r-s) !}{(r-2 s) !} \\
\operatorname{vol}_{G^{(s)}}\left(\bar{T}^{\prime}\right) & =\operatorname{vol}(\bar{T}) \frac{t !(r-t) !}{(r-2 s) !}
\end{aligned}
$$

Let $E_{G^{(s)}}\left(S^{\prime}, T^{\prime}\right)$ be the number of edges between $S^{\prime}$ and $T^{\prime}$ in $G^{(s)}$. We get

$$
\left|E_{G^{(s)}}\left(S^{\prime}, T^{\prime}\right)\right|=\frac{(r-s-t) ! s ! t !}{(r-2 s) !}|E(S, T)| .
$$

Applying Theorem 2.4 to the sets $S^{\prime}$ and $T^{\prime}$ in $G^{(s)}$, we obtain

$$
\begin{aligned}
& || E_{G^{(s)}}\left(S^{\prime}, T^{\prime}\right)\left|-\frac{\operatorname{vol}_{G^{(s)}}\left(S^{\prime}\right) \operatorname{vol}_{G^{(s)}}\left(T^{\prime}\right)}{\operatorname{vol}_{G^{(s)}}\left(G^{(s)}\right)}\right| \\
& \quad \leq \bar{\lambda}_{1}^{(s)} \frac{\sqrt{\operatorname{vol}_{G^{(s)}}\left(S^{\prime}\right) \operatorname{vol}_{G^{(s)}}\left(T^{\prime}\right) \operatorname{vol}_{G^{(s)}}\left(\bar{S}^{\prime}\right) \operatorname{vol}_{G^{(s)}}\left(\bar{T}^{\prime}\right)}}{\operatorname{vol}_{G^{(s)}}\left(G^{(s)}\right)} .
\end{aligned}
$$

Combining (5.2) through (5.6) and the inequality above, we obtain inequality (5.1).

Now we consider the case that $s>r / 2$. Due to the fact that $\sigma_{0}^{(s)}=1$, we have to use the weaker expansion theorem, Theorem 2.9. Note that

$$
\left|E\left(\left(\begin{array}{l}
V \\
s
\end{array}\right),\left(\begin{array}{l}
V \\
t
\end{array}\right)\right)\right|=|E(H)| \frac{r !}{(r-s-t) ! s ! t !} .
$$

We get the following theorem.

Theorem 5.10. For $1 \leq t<r / 2<s<s+t \leq r, S \subseteq\left(\begin{array}{c}V \\ s\end{array}\right)$, and $T \subseteq\left(\begin{array}{c}V \\ t\end{array}\right)$, let

$$
e(S, T)=\frac{|E(S, T)|}{\left|E\left(\left(\begin{array}{c}
V \\
s
\end{array}\right),\left(\begin{array}{c}
V \\
t
\end{array}\right)\right)\right|} .
$$

If $|x \cap y| \neq \min \{t, 2 s-r\}$ for every $x \in S$ and $y \in T$, then we have

$$
\left|\frac{1}{2} e(S, T)-e(S) e(T)\right| \leq \bar{\lambda}^{(s)} \sqrt{e(S) e(T) e(\bar{S}) e(\bar{T})} .
$$


Proof. Recall that $D^{(s)}$ is the directed graph defined in Section 3. Let

$$
\begin{aligned}
& S^{\prime}=\left\{x \in \mathrm{V}^{\underline{s}} \mid[x] \in S\right\}, \\
& T^{\prime}=\left\{(y, z) \in \mathrm{V}^{\underline{s}} \mid[z] \in T\right\} .
\end{aligned}
$$

We also denote by $\bar{S}^{\prime}$ and $\bar{T}^{\prime}$ the respective complements of $S^{\prime}$ and $T^{\prime}$ in $\mathrm{V}^{\underline{s}}$. We use the convention that $\operatorname{vol}_{D^{(s)}}(*)$ denotes volumes in $D^{(s)}$, while $\operatorname{vol}(*)$ denotes volumes in the hypergraph $H$. We have

$$
\begin{aligned}
\operatorname{vol}_{D^{(s)}}\left(D^{(s)}\right) & =\operatorname{vol}\left(\left(\begin{array}{c}
V \\
s
\end{array}\right)\right) s !(r-s) !, \\
\operatorname{vol}_{D^{(s)}}\left(S^{\prime}\right) & =\operatorname{vol}(S) s !(r-s) ! \\
\operatorname{vol}_{D^{(s)}}\left(T^{\prime}\right) & =\operatorname{vol}(T) t !(r-t) ! \\
\operatorname{vol}_{D^{(s)}}\left(\bar{S}^{\prime}\right) & =\operatorname{vol}(\bar{S}) s !(r-s) ! \\
\operatorname{vol}_{D^{(s)}}\left(\bar{T}^{\prime}\right) & =\operatorname{vol}(\bar{T}) s !(r-s) !
\end{aligned}
$$

Let $E_{D^{(s)}}\left(S^{\prime}, T^{\prime}\right)$ be the number of directed edges from $S^{\prime}$ to $T^{\prime}$ in $D^{(s)}$, and let $E_{D^{(s)}}\left(T^{\prime}, S^{\prime}\right)$ be the number of such edges from $T^{\prime}$ to $S^{\prime}$. We get

$$
\left|E_{D^{(s)}}\left(S^{\prime}, T^{\prime}\right)\right|=(r-s-t) ! s ! t !|E(S, T)| .
$$

From the condition $|x \cap y| \neq \min \{t, 2 s-r\}$ for each $x \in S$ and each $y \in T$, we observe that

$$
E_{D^{(s)}}\left(T^{\prime}, S^{\prime}\right)=0 .
$$

Applying Theorem 2.9 to the sets $S^{\prime}$ and $T^{\prime}$ in $D^{(s)}$, we obtain

$$
\begin{gathered}
\left|\frac{\left|E_{D^{(s)}}\left(S^{\prime}, T^{\prime}\right)\right|+\left|E_{D^{(s)}}\left(T^{\prime}, S^{\prime}\right)\right|}{2}-\frac{\operatorname{vol}_{D^{(s)}}\left(S^{\prime}\right) \operatorname{vol}_{D^{(s)}}\left(T^{\prime}\right)}{\operatorname{vol}_{D^{(s)}}\left(D^{(s)}\right)}\right| \\
\leq \bar{\lambda}_{1}^{(s)} \frac{\sqrt{\operatorname{vol}_{D^{(s)}}\left(S^{\prime}\right) \operatorname{vol}_{D^{(s)}}\left(T^{\prime}\right) \operatorname{vol}_{D^{(s)}}\left(\bar{S}^{\prime}\right) \operatorname{vol}_{D^{(s)}}\left(\bar{T}^{\prime}\right)}}{\operatorname{vol}_{D^{(s)}}\left(D^{(s)}\right)}
\end{gathered}
$$

Combining (5.8) through (5.12) and the inequality above, we get inequality (5.7).

Nevertheless, we have the following strong edge expansion theorem for $r / 2<$ $s \leq r-1$. For $S, T \subseteq\left(\begin{array}{c}V \\ s\end{array}\right)$, let $E^{\prime}(S, T)$ be the set of edges of the form $x \cup y$ for some $x \in S$ and $y \in T$. Namely,

$$
E^{\prime}(S, T)=\{F \in E(H) \mid \exists x \in S, \exists y \in T, F=x \cup y\} .
$$

Observe that

$$
\left|E^{\prime}\left(\left(\begin{array}{l}
V \\
s
\end{array}\right),\left(\begin{array}{l}
V \\
s
\end{array}\right)\right)\right|=|E(H)| \frac{r !}{(r-s) !(2 s-r) !(r-s) !} .
$$


Theorem 5.II. For $r / 2<s \leq r-1$ and $S, T \subseteq\left(\begin{array}{c}V \\ s\end{array}\right)$, let

$$
e^{\prime}(S, T)=\frac{\left|E^{\prime}(S, T)\right|}{\left|E^{\prime}\left(\left(\begin{array}{l}
V \\
s
\end{array}\right),\left(\begin{array}{l}
V \\
s
\end{array}\right)\right)\right|} .
$$

We have

$$
\left|e^{\prime}(S, T)-e(S) e(T)\right| \leq \bar{\lambda}^{(s)} \sqrt{e(S) e(T) e(\bar{S}) e(\bar{T})}
$$

Proof. Let

$$
\begin{aligned}
& S^{\prime}=\left\{x \in \mathrm{V}^{\underline{s}} \mid[x] \in S\right\}, \\
& T^{\prime}=\left\{y \in \mathrm{V}^{\underline{s}} \mid[y[\in T\} .\right.
\end{aligned}
$$

Let $\bar{S}^{\prime}$ and $\bar{T}^{\prime}$ be the respective complements of $S^{\prime}$ and $T^{\prime}$ in $\mathrm{V}^{\underline{s}}$. We use the convention that $\operatorname{vol}_{D^{(s)}}(*)$ denotes volumes in $D^{(s)}$, while $\operatorname{vol}(*)$ denotes volumes in the hypergraph $H$. We have

$$
\begin{aligned}
\operatorname{vol}_{D^{(s)}}\left(D^{(s)}\right) & =\operatorname{vol}\left(\left(\begin{array}{c}
V \\
s
\end{array}\right)\right) s !(r-s) ! ; \\
\operatorname{vol}_{D^{(s)}}\left(S^{\prime}\right) & =\operatorname{vol}(S) s !(r-s) ! ; \\
\operatorname{vol}_{D^{(s)}}\left(T^{\prime}\right) & =\operatorname{vol}(T) s !(r-s) ! \\
\operatorname{vol}_{D^{(s)}}\left(\bar{S}^{\prime}\right) & =\operatorname{vol}(\bar{S}) s !(r-s) ! \\
\operatorname{vol}_{D^{(s)}}\left(\bar{T}^{\prime}\right) & =\operatorname{vol}(\bar{T}) s !(r-s) !
\end{aligned}
$$

Let $E_{D^{(s)}}\left(S^{\prime}, T^{\prime}\right)$ and $E_{D^{(s)}}\left(T^{\prime}, S^{\prime}\right)$ be the respective numbers of directed edges from $S^{\prime}$ to $T^{\prime}$ and from $T^{\prime}$ to $S^{\prime}$ in $D^{(s)}$. We get

$$
\left|E_{D^{(s)}}\left(S^{\prime}, T^{\prime}\right)\right|=\left|E_{D^{(s)}}\left(T^{\prime}, S^{\prime}\right)\right|=(r-s) !(2 s-r) !(r-s) !\left|E^{\prime}(S, T)\right| .
$$

Applying Theorem 2.9 to the sets $S^{\prime}$ and $T^{\prime}$ on $D^{(s)}$, we obtain

$$
\begin{gathered}
\left|\frac{\left|E_{D^{(s)}}\left(S^{\prime}, T^{\prime}\right)\right|+\left|E_{D^{(s)}}\left(T^{\prime}, S^{\prime}\right)\right|}{2}-\frac{\operatorname{vol}_{D^{(s)}}\left(S^{\prime}\right) \operatorname{vol}_{D^{(s)}}\left(T^{\prime}\right)}{\operatorname{vol}_{D^{(s)}}\left(D^{(s)}\right)}\right| \\
\leq \bar{\lambda}_{1}^{(s)} \frac{\sqrt{\operatorname{vol}_{D^{(s)}}\left(S^{\prime}\right) \operatorname{vol}_{D^{(s)}}\left(T^{\prime}\right) \operatorname{vol}_{D^{(s)}}\left(\bar{S}^{\prime}\right) \operatorname{vol}_{D^{(s)}}\left(\bar{T}^{\prime}\right)}}{\operatorname{vol}_{D^{(s)}}\left(D^{(s)}\right)} .
\end{gathered}
$$

Combining (5.14) through (5.18) and the inequality above, we get inequality (5.13).

\section{Concluding Remarks}

In this paper, we introduced a set of Laplacians for $r$-uniform hypergraphs. For $1 \leq s \leq r-1$, the $s$-Laplacian $\mathcal{L}^{(s)}$ is derived from the random $s$-walks on 
hypergraphs. For $1 \leq s \leq r / 2$, the $s$ th Laplacian $\mathcal{L}^{(s)}$ is defined to be the Laplacian of the corresponding weighted graph $G^{(s)}$. The first Laplacian $\mathcal{L}^{(1)}$ is exactly the Laplacian introduced in [Rodríguez 09].

For $r / 2 \leq s \leq r-1$, the $s$ th Laplacian $\mathcal{L}^{(s)}$ is defined to be the Laplacian of the corresponding Eulerian directed graph $D^{(s)}$. From Lemma 2.6, Theorem 2.7, and Theorem 2.8, it seems that $\sigma_{0}\left(D^{(s)}\right)$ might be a good parameter. However, it is not hard to show that $\sigma_{0}\left(D^{(s)}\right)=1$ always holds, which makes Theorem 2.8 useless for hypergraphs. We can use the weaker Theorem 2.9 for hypergraphs. Our work is based on (with some improvements) the recent work [Chung 05, Chung 06] on directed graphs.

Let us recall Chung's definition of Laplacians [Chung 93] for regular hypergraphs. An $r$-uniform hypergraph $H$ is $d$-regular if $d_{x}=d$ for every $x \in \mathrm{V} \underline{r-1}$. Let $G$ be a graph on the vertex set $\mathrm{V}^{\underline{r-1}}$. For $x, y \in \mathrm{V}^{\underline{r-1}}$, let $x y$ be an edge if $x=x_{1} x_{2}, \ldots, x_{r-1}$ and $y=y_{1} x_{2}, \ldots, x_{r-1}$ such that $\left\{x_{1}, y_{1}, x_{2}, \ldots, x_{r-1}\right\}$ is an edge of $H$. Let $A$ be the adjacency matrix of $G, T$ the diagonal matrix of degrees in $G$, and $K$ the adjacency matrix of the complete graph on the edge set $\mathrm{V} \stackrel{r-1}{\text {. }}$ In [Chung 93], the Laplacian $\mathcal{L}$ is defined such that

$$
\mathcal{L}=T-A+\frac{d}{n}(K+(r-1) I)
$$

This definition comes from the homology theory of hypergraphs [Chung 93]. Firstly, $\mathcal{L}$ is not normalized in Chung's definition, i.e., the eigenvalues are not in the interval $[0,2]$. Secondly, the add-on term

$$
\frac{d}{n}(K+(r-1) I)
$$

is not related to the structures of $H$. If we ignore the add-on term and normalize the matrix, then we essentially get the Laplacian of the graph $G$. Note that if $G$ is disconnected, then $\lambda_{1}(G)=0$, and this situation is not interesting. Thus Chung added an additional term. The graph $G$ is actually very close to our Eulerian directed graph $D^{(r-1)}$. Let $B$ be the adjacency matrix of $D^{(r-1)}$. In fact, we have $B=Q A$, where $Q$ is a rotation that maps $x=x_{1}, x_{2}, \ldots, x_{r-1}$ to $x^{\prime}=x_{2}, \ldots, x_{r-1}, x_{1}$. Since $d_{x}=d_{x^{\prime}}$, it follows that $Q$ and $T$ commute, and we have

$$
\begin{aligned}
& \left(T^{-1 / 2} B T^{-1 / 2}\right)^{\prime}\left(T^{-1 / 2} B T^{-1 / 2}\right)=T^{-1 / 2} B^{\prime} T^{-1} B T^{-1 / 2} \\
& \quad=T^{-1 / 2} A^{\prime} Q^{\prime} T^{-1} Q A T^{-1 / 2}=T^{-1 / 2} A^{\prime} T^{-1} Q^{\prime} Q A T^{-1 / 2} \\
& \quad=T^{-1 / 2} A^{\prime} T^{-1} A T^{-1 / 2} .
\end{aligned}
$$


Here we use the fact that $Q^{\prime} Q=I$. This identity means that the singular values of $I-\mathcal{L}^{(r-1)}$ are precisely equal to 1 minus the Laplacian eigenvalues of the graph $G$.

Our definitions of Laplacians $\mathcal{L}^{(s)}$ seem to be related to the quasirandomness [Chung and Graham 90, Kohayakawa et al. 02] of hypergraphs. We are very interested in this direction. Many concepts such as the $s$-walk, the $s$-path, the $s$-distance, and the $s$-diameter are of independent interest.

Acknowledgments. Linyuan Lu was supported in part by NSF grant DMS 1000475. Xing Peng was supported in part by NSF grant DMS 1000475.

\section{References}

[Aldous and Fill 12] D. Aldous and J. Fill. Reversible Markov Chains and Random Walks on Graphs. In preparation, 2012.

[Alon 86] N. Alon. "Eigenvalues and Expanders." Combinatorica 6 (1986), 86-96.

[Butler unpubl.] S. Butler. "A New Discrepancy Definition for Hypergraphs." Available online (http://www.math.ucsd.edu/ sbutler/PDF/hyprdisc.pdf).

[Chung 89] F. Chung. "Diameters and Eigenvalues." J. of the Amer. Math. Soc. 2 (1989), 187-196.

[Chung 93] F. Chung. "The Laplacian of a Hypergraph." In Expanding Graphs, edited by J. Friedman, DIMACS series, pp. 21-36. AMS, 1993.

[Chung 97] F. Chung. Spectral Graph Theory. AMS publications, 1997.

[Chung 05] F. Chung. "Laplacians and the Cheeger Inequality for Directed Graphs." Annals of Comb. 9 (2005), 1-19.

[Chung 06] F. Chung. "The Diameter and Laplacian Eigenvalues of Directed Graphs." Electronic Journal of Combinatorics 13 (2006), N4.

[Chung and Graham 90] F. Chung and R. L. Graham. "Quasi-random Hypergraphs." Random Structure and Algorithms 1:1 (1990), 105-124.

[Chung et al. 94] F. Chung, V. Faber, and T. A. Manteuffel. "An Upper Bound on the Diameter of a Graph from Eigenvalues Associated with Its Laplacian." SIAM. J. Disc. Math 7:3 (1994), 443-457.

[Cooper and Dutle 12] J. Cooper and A. Dutle. "Spectra of Uniform Hypergraphs." Linear Algebra and Its Applications 436:9 (2012), 3268-3292.

[Demir et al. 08] E. Demir, C. Aykanat, and B. B. Cambazoglu. "Clustering Spatial Networks for Aggregate Query Processing: A Hypergraph Approach." Information Systems 33:1 (2008), 1-17.

[Dudek and Frieze 11] A. Dudek and A. M. Frieze. "Loose Hamilton Cycles in Random Uniform Hypergraphs." Electronic Journal of Combinatorics 18:1 (2011), P48. 
[Dudek and Frieze 12] A. Dudek and A. M. Frieze. "Tight Hamilton Cycles in Random Uniform Hypergraphs." Random Structures and Algorithms. Preprint available online (http://onlinelibrary.wiley.com/doi/10.1002/rsa.20404/full).

[Friedman and Wigderson 95] J. Friedman and A. Wigderson. "On the Second Eigenvalue of Hypergraphs." Combinatorica 15:1 (1995), 43-65.

[Frieze 10] A. M. Frieze. "Loose Hamilton Cycles in Random 3-Uniform Hypergraphs." Electronic Journal of Combinatorics 17 (2010), N28.

[Hàn and Schacht 99] H. Hàn and M. Schacht. "Dirac-Type Results for Loose Hamilton Cycles in Uniform Hypergraphs." J. Comb. Theory Ser. B 100 (2010), 332-346.

[Katona and Kierstead 99] G. Y. Katona and H. A. Kierstead. "Hamiltonian Chains in Hypergraphs." J. of. Graph Theory 30:3 (1999), 205-212.

[Keevash et al. 12] P. Keevash, D. Kühn, R. Mycroft, and D. Osthus. "Loose Hamilton Cycles in Hypergraphs." To appear, 2012.

[Klamt et al. 09] S. Klamt, U.-U. Haus, and F. Theis. "Hypergraphs and Cellular Networks." PLoS Comput Biol 5:5 (2009), e1000385.

[Kohayakawa et al. 02] Y. Kohayakawa, V. Rödl, and J. Skokan. "Hypergraphs, Quasirandomness, and Conditions for Regularity." J. Combin. Theory Ser. A 97:2 (2002), $307-352$.

[Kühn and Osthus 06] D. Kühn and D. Osthus. "Loose Hamilton Cycles in 3-Unifrom Hypergraphs of High Minimum Degree." J. Combin. Theory Ser. B 96:6 (2006), $767-821$.

[Kühn et al. 12] D. Kühn, R. Mycroft, and D. Osthus. "Hamilton l-Cycles in $k$-Graphs." To appear, 2012.

[Lawler and Sokal 88] G. F. Lawler and A. D. Sokal. "Bounds on the $L^{2}$ Spectrum for Markov Chains and Markov Processes: A Generalization of Cheeger's Inequality." Transactions of the American Mathematical Society, 309 (1988), 557-580.

[Li 04] W.-C. W. Li. "Ramanujan Hypergraphs." Geom. Funct. Anal., 14:2 (2004), 380-399.

[Li and Solé 96] W.-C. W. Li and P. Solé. "Spectra of Regular Graphs and Hypergraphs and Orthogonal Polynomials." Europ. J. Combinatorics 17 (1996), 461-477.

[Mihail 89] M. Mihail. "Conductance and Convergence of Markov Chains: A Combinatorial Treatment of Expanders." In Proc. of 30th FOCS, pp. 526-531, 1989.

[Rödl et al. 06] V. Rödl, A. Ruciński, and E. Szemerédi. "A Dirac-Type Theorem for 3-Uniform Hypergraphs." Combin. Probab. Comput, 15:1-2 (2006), 229-251.

[Rödl et al. 08] V. Rödl, A. Ruciński, and E. Szemerédi. "An Approximate Dirac-Type Theorem for $k$-Uniform Hypergraphs." Combinatorica 28:2 (2008), 229-260.

[Rodríguez 09] J. A. Rodríguez. "Laplacian Eigenvalues and Partition Problems in Hypergraphs." Applied Mathematics Letters 22 (2009) 916-921.

[Zhang 07] B.-T. Zhang. "Random Hypergraph Models of Learning and Memory in Biomolecular Networks: Shorter-Term Adaptability vs. Longer-Term Persistency." In IEEE Symposium on Foundations of Computational Intelligence (FOCI 2007), pp. 344-349, 2007. 
Linyuan Lu, Department of Mathematics, University of South Carolina, Columbia, SC 29208 (lu@math.sc.edu)

Xing Peng, Department of Mathematics, University of South Carolina, Columbia, SC 29208 (pengx@mailbox.sc.edu) 